\title{
Resistência ao fogo em uma população de Cyathea atrovirens (Langsd. \& Fisch.) Domin (Cyatheaceae) no Estado do Rio Grande do Sul, Brasil
}

\author{
Carlos Rodrigo Lehn ${ }^{1,3 *}$ \\ Caroline Leuchtenberger ${ }^{2,3}$ \\ ${ }^{1} \mathrm{PPG}$ em Biologia Vegetal, Universidade Federal de Mato Grosso do Sul \\ ${ }^{2}$ PPG Ecologia e Conservação, Universidade Federal de Mato Grosso do Sul \\ ${ }^{3}$ Embrapa Pantanal, Caixa Postal 109, CEP 79320-900, Corumbá - MS, Brasil \\ *Autor para correspondência \\ crlehn@gmail.com
}

Submetido em 06/09/2007

Aceito para publicação em 09/06/2008

\section{Resumo}

Cyathea atrovirens (Langsd. \& Fisch.) Domin é uma samambaia arborescente que no Estado do Rio Grande do Sul ocorre desde o nível do mar até áreas com aproximadamente $300 \mathrm{~m}$ de altitude. Monitoramos uma população de $C$. atrovirens formada por 10 indivíduos, atingida por uma queimada, no município de Campo Bom, Rio Grande do Sul, Brasil. A população estudada situa-se em local desprovido de vegetação, em um barranco úmido à beira do $\mathrm{km} 11$ da rodovia RS-239. Os objetivos do estudo foram analisar as respostas da população após a passagem do fogo, em relação aos seguintes aspectos: produção de novas frondes (vegetativas e férteis); taxa anual de crescimento relativo do comprimento do cáudice, bem como a produção, maturação e liberação dos esporos. Quanto maior o esporófito, maior tende a ser a produção de frondes. Observamos uma relação significativa entre comprimento dos cáudices e taxa de crescimento relativo $\left(\mathrm{T}_{\mathrm{CR}}\right)$. Ao longo do primeiro ano após a queimada, todos os indivíduos observados formaram frondes férteis, de modo assincrônico. A espécie mostrou-se capaz de suportar a passagem rápida de fogo, uma vez que o restabelecimento na produção de frondes (vegetativas e férteis) ocorreu gradualmente após a queimada.

Unitermos: frondes, queimada, samambaia arborescente

\section{Abstract}

Fire resistance in a population of Cyathea atrovirens (Langsd. \& Fisch.) Domin (Cyatheaceae) in the state of Rio Grande do Sul, Brazil. Cyathea atrovirens (Langsd. \& Fisch.) Domin is a tree fern that occurs in the state of Rio Grande do Sul from sea level to an altitude of $300 \mathrm{~m}$. We monitored a population of 10 individuals of $C$. atrovirens affected by burning in the municipality of Campo Bom, Rio Grande do Sul, Brazil. The objective of this study was to analyze the responses of a Cyathea atrovirens population after the occurrence of a fire, considering the following aspects: frond production, annual rates of relative growth of caudex length, and the production, maturation and release of spores. The sporophytes of larger size produced more fronds than the smaller ones. We observed a significant relationship between caudex length and relative growth $\left(\mathrm{T}_{\mathrm{CR}}\right)$ in individuals of smaller size. In the first year, after the fire, all individuals produced new fronds. The spore production was asynchronic. The species apparently demonstrated its capacity to support the occurrence of quick fires, since the post-fire frond production was gradually reestablished.

Key words: fronds, fire, tree fern 


\section{Introdução}

A mudança na abundância de uma espécie pode potencialmente ter um efeito mais sério no ecossistema, quando essa alteração ocorre em espécies raras (Ough e Murphy, 2004). A ocorrência de condições extremas experimentadas em intervalos regulares ou irregulares de tempo pode servir como restrição significante na ecologia das espécies, se elas não apresentarem adaptações contra isto. Entre os diversos fatores que podem alterar a abundância de uma espécie ou população está a ação de queimadas (Pinnard e Hufmann, 1997).

O fogo é considerado por muitos autores como um dos mais poderosos fatores ambientais, que podem moldar a vegetação, principalmente em áreas sazonalmente secas (Gignoux et al., 1997; Felfili et al., 1999) e em regiões de clima mesotérmico que podem temporariamente estar passando por um período de seca prolongada. Até onde se é sabido, não existem estudos realizados sobre a capacidade que as pteridófitas possuem de responder aos danos causados pelo fogo na região sul do Brasil. Embora estudos pioneiros tenham sido realizados na África por Kornás (1975, 1978, 1979 e 1993), ainda hoje existe uma carência muito grande de trabalhos que abordem este tema.

As ciateáceas são pteridófitas escamosas que têm hábito usualmente arborescente, consistindo de um cáudice simples com uma roseta de frondes no ápice (Fernandes, 2003). A família possui distribuição pantropical, sendo verificada a ocorrência de todos os gêneros nos trópicos americanos. Alsophila e Sphaeropteris também ocorrem nos paleotrópicos, com maior concentração de espécies em regiões montanhosas (Tryon, 1970). No Brasil, a família possui representantes citados para todas as regiões (Fernandes, 1997 e 2003; Windisch e Tryon, 2001; Prado e Freitas, 2005). As samambaias arborescentes são um importante componente em formações florestais situadas em áreas de média e alta elevação na América do Sul (Arens, 1998). Dentro dessas formações, os representantes da família ocupam uma ampla variedade de ambientes, entre os quais florestas primárias, florestas secundárias ou em regeneração, bem como áreas abertas como pastagens abandonadas (Tryon e Tryon, 1982).
A fim de se verificar a tolerância de uma samambaia arborescente com relação à passagem do fogo, monitoramos as respostas de uma população de Cyathea atrovirens (Langsd. \& Fisch.) Domin atingida por uma queimada, em relação aos seguintes aspectos: 1) produção de novas frondes (vegetativas e férteis); 2) taxa anual de crescimento relativo do comprimento do cáudice e 3) produção, maturação e liberação dos esporos.

\section{Material e Métodos}

Cyathea atrovirens (Langsd \& Fisch) Domin caracteriza-se por apresentar um cáudice ereto que pode ultrapassar $5 \mathrm{~m}$ de comprimento, envolto em sua base por uma camada de raízes adventícias (Fernandes, 2000). A espécie apresenta valor comercial, sendo amplamente utilizada na região de estudo para fins paisagísticos.

No Estado do Rio Grande do Sul Cyathea atrovirens é encontrada desde a Planície Litorânea até áreas com cerca de 300m de altitude (Sehnem, 1978), sendo que apenas uma coleta foi verificada para o município de Cambará do Sul, situado a aproximadamente $950 \mathrm{~m}$ acima do nível do mar (Lorscheitter et al., 1998). A espécie cresce em locais abertos, sujeitos à incidência solar plena, bem como em locais moderadamente sombreados. Populações relativamente significativas da espécie são encontradas em áreas de mata paludosa, barrancos e valas situadas às margens de estradas e rodovias (C. R. Lehn, observação pessoal).

A população estudada encontra-se situada junto ao $\mathrm{km} 11$ da rodovia RS-239 (29 82'24"'S e 51'22'17'”W), no município de Campo Bom-RS, crescendo junto a um barranco úmido, desprovido completamente de vegetação, estando bastante exposta à incidência solar.

Monitoramos todos os dez indivíduos da população, identificados por um número em uma placa de alumínio, presa a uma pequena estaca de madeira junto à base dos esporófitos. Cada cáudice aéreo foi considerado um indivíduo. As coletas de dados foram realizadas mensalmente, entre abril de 2004 e março de 2005. A passagem do fogo se deu entre os dias 21 e 22 de abril de 2004 
A taxa anual de novas frondes correspondeu ao número total de báculos por planta que se expandiram, originando novas frondes. Para avaliar a capacidade de resposta da espécie em relação à passagem do fogo, comparamos os dados referentes à produção anual de frondes com dados disponíveis na literatura sobre espécies de hábito semelhante e com outra população da referida espécie (Schmitt e Windisch, 2006a).

Para o cálculo da taxa anual de crescimento relativo do comprimento do cáudice $\left(\mathrm{T}_{\mathrm{CR}}\right)$ utilizamos a seguinte fórmula:

$$
\mathrm{T}_{\mathrm{CR}}=\left(\mathrm{L}_{2} / \mathrm{L}_{1}\right)-1 \times 100 \text {, onde: }
$$

$\mathrm{T}_{\mathrm{CR}}=$ taxa de crescimento relativo do comprimento do cáudice em $\%$.

$\mathrm{L}_{1}=$ medida do comprimento do cáudice, em centímetros, no primeiro levantamento.

$\mathrm{L}_{2}=$ medida do comprimento do cáudice, em centímetros, no segundo levantamento.

A produção, maturação e liberação dos esporos foram acompanhadas em todas as visitas à população. $\mathrm{O}$ início da liberação foi determinado a partir do momento em que os esporângios apresentaram sinais de abertura. Dados climatológicos foram obtidos junto a Estação Meteorológica do município de Campo Bom-RS.

No presente trabalho foi adotado o sistema de classificação de Lellinger (1987). Os termos cáudice, estípite e fronde, específicos da pteridologia, correspondem, respectivamente, a caule, pecíolo e folha, das angiospermas. Para se analisar a relação entre comprimento do cáudice e produção anual de frondes, bem como a relação entre comprimento do cáudice e taxa crescimento relativo dos cáudices, utilizamos a análise de regressão linear simples, segundo métodos descritos em Zar (1999). A análise dos dados foi realizada utilizando-se o programa SYSTAT, versão 11 (Wilkinson, 2004).

\section{Resultados e Discussão}

Todos os indivíduos tiveram perda total de frondes após a ocorrência da queimada, não sendo observados báculos remanescentes à queimada (Figura 1).

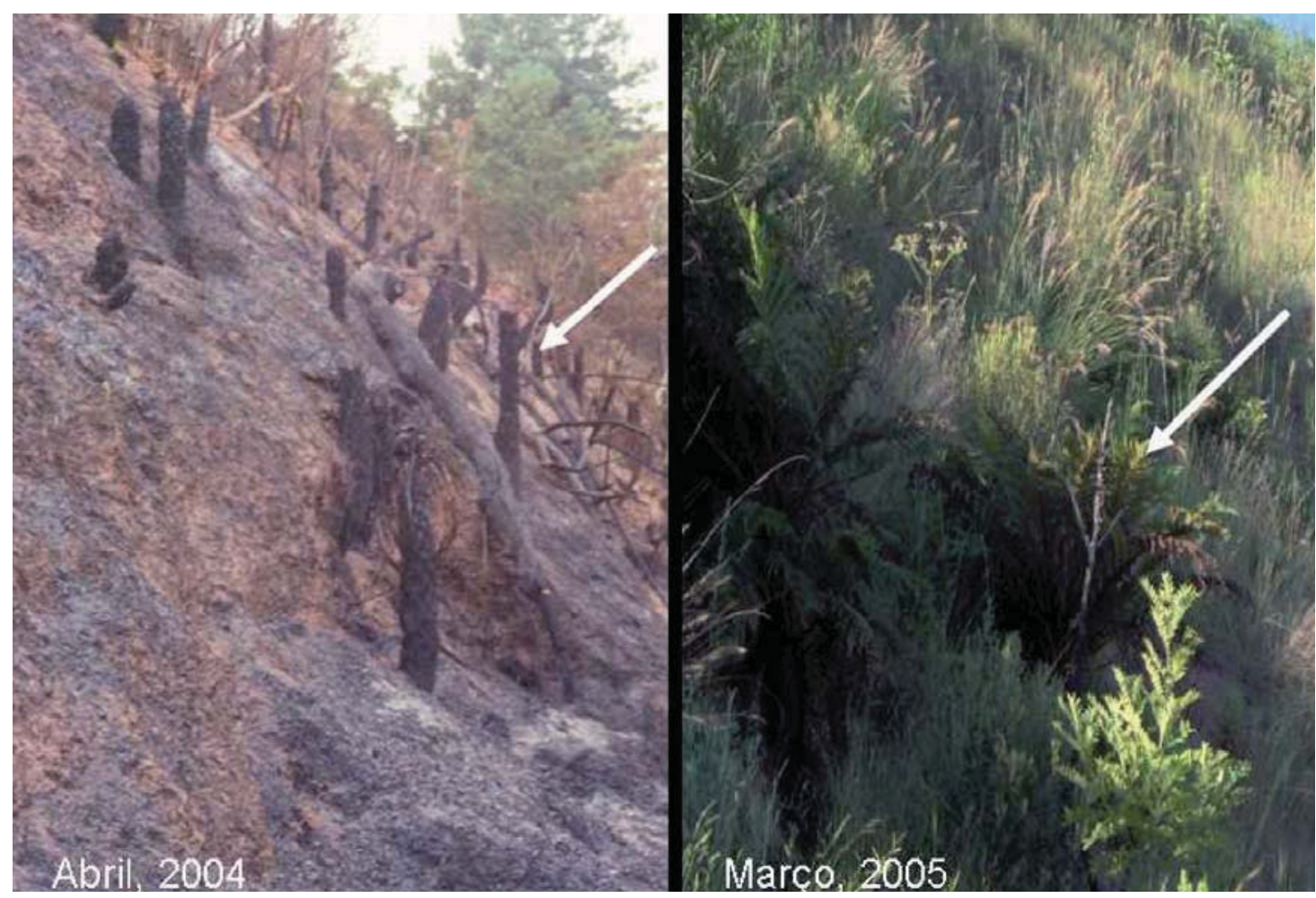

FIGURA 1: População de Cyathea atrovirens (Langsd. \& Fisch.) Domin situada no município de Campo Bom/RS, Brasil, logo após a passagem do fogo em abril de 2004 e em março de 2005, no final do período de observações. 
Durante o estudo nenhum esporófito analisado produziu frondes senescentes. Sendo assim, o número de frondes observadas junto aos esporófitos em março de 2005, corresponde à taxa anual de produção de novas frondes por indivíduo, na população estudada. Cabe ressaltar que as primeiras frondes férteis que apresentaram sinais de maturação e liberação de esporos, ao final do estudo (março-2005) já mostravam sinais de senescência. Diversos autores reportam longevidade prolongada para frondes de pteridófitas arborescentes (Tanner, 1983; Schmitt, 2001) em populações situadas no interior de florestas. Entretanto, como na população estudada as frondes estão expostas diretamente às condições de ressecamento (incidência solar, geadas, frio) é bem provável que as frondes vegetativas não apresentem longevidade muito acentuada.

Todos os indivíduos formaram novas frondes entre maio e julho (2004). No intervalo compreendido entre os meses de agosto e outubro/2004, nenhum indivíduo formou novas frondes. No mês de novembro observamos um pico na produção de novas frondes, precedido de um período de elevada pluviosidade (Figura 2). Corroborando com o presente estudo, outros autores reportam que o aumento da pluviosidade pode estimular a produção de frondes em pteridófitas arborescentes (Cyathea pubescens Mett. ex Kuhn - Tanner, 1983; C. caracasana (Klotzsch) Domin -Arens, 2001; Cibotium taiwanense C.M.Kuo - Chiou et al., 2001, Alsophila setosa Kaulf-Schmitt e Windisch, 2006b). Ainda assim, não observamos relação entre pluviosidade e produção de frondes $(\mathrm{P}=0,79)$.

Durante o primeiro ano após a queimada, os esporófitos de maior tamanho produziram um número maior de frondes, com um máximo de 14, para o maior esporófito observado $(154 \mathrm{~cm})$. O esporófito de menor tamanho $(67 \mathrm{~cm})$ foi também o responsável pela produção do menor número de frondes $(\mathrm{n}=7)$. A análise desses dados revelou uma relação forte e positiva entre produção anual de frondes e comprimento dos cáudices $\left(\mathrm{R}^{2}=0,95 ; \mathrm{P}<0,001\right)$ (Figura 3). Corroborando com os dados obtidos no presente estudo, Schmitt e Windisch (2003) para duas populações de Alsophila setosa e Tanner (1983) para Cyathea pubescens registraram a ocorrência de uma maior produção de frondes nos esporófitos de maior tamanho. Entretanto, Seiler (1981) observou uma produção constante de frondes não relacionada ao tamanho do cáudice em Alsophila salvinii Hook.
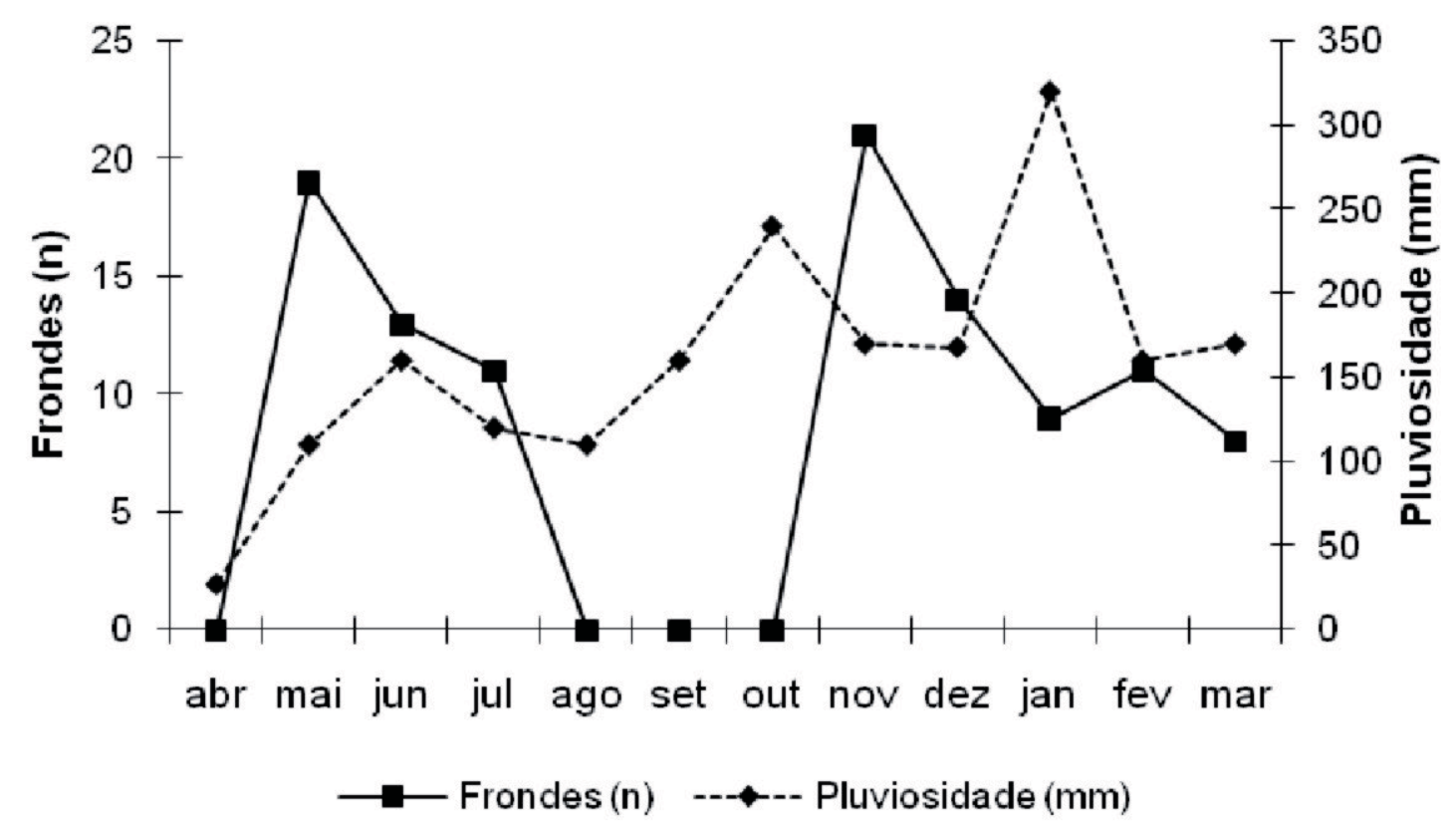

FIGURA 2: Sazonalidade da produção de frondes entre abril/2004 e março/2005 para uma população Cyathea atrovirens (Langsd. \& Fisch.) Domin, situada no município de Campo Bom, estado do Rio Grande do Sul, Brasil. 
O número médio de frondes na população é semelhante ao número médio de frondes encontrado em outras espécies de hábito semelhante, bem como para outra população da referida espécie (Tabela 1).

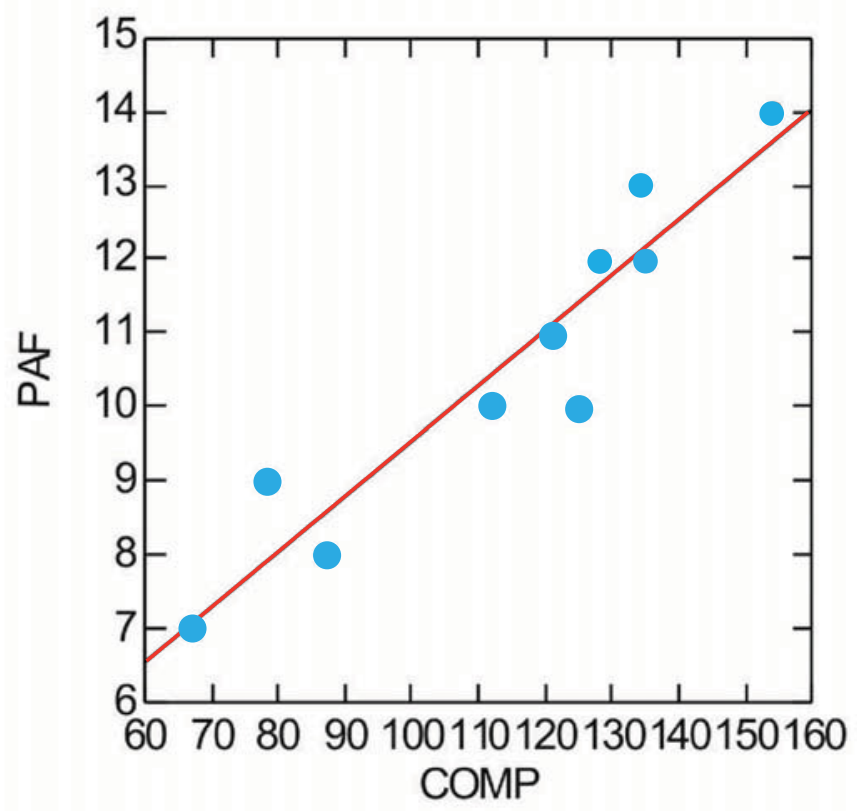

FIGURA 3: Relação entre comprimento do cáudice (COMP/cm) e produção de novas frondes (PAF), para uma população Cyathea atrovirens (Langsd. \& Fisch.) Domin, situada no município de Campo Bom/RS, Brasil.

A produção e maturação dos esporos ocorreram de maneira assincrônica, sendo o período de maturação dos esporos completado em até cinco meses. Os esporos de cinco frondes férteis amadureceram entre julho e outubro 2004. Para outras 10 frondes os esporos amadureceram em novembro 2004 e fevereiro 2005. O início da liberação dos esporos ocorreu de forma gradual. Enquanto que, em uma fronde a liberação teve início em aproximadamente quatro meses após a formação das frondes férteis, na grande maioria das frondes a liberação teve início a partir do quinto mês após o surgimento da fronde fértil. Outro aspecto interessante, é que a liberação dos esporos ocorre de forma gradual dentro de uma mesma fronde, tendo início nas pinas basais.

A assincronia na liberação dos esporos observada neste estudo pode ser um fator positivo para a população, uma vez que, em caso de haver algum período desfavorável para germinação dos esporos e estabelecimento dos gametófitos, evita que toda a produção seja perdida. Tal assincronia pode ser verificada para algumas espécies pertencentes às famílias Polypodiaceae e Pteridaceae (Ranal, 1995), como também para Alsophila setosa (Schmitt e Windisch, 2006b) e para Danaea sellowiana C.Presl (Lehn, 2008).

TABELA 1: Número médio de frondes/ano por esporófito. ${ }^{1}$ Presente estudo; ${ }^{2} \mathrm{Schmitt}$ e Windisch, 2006a; ${ }^{3}$ Tanner, 1983; ${ }^{4}$ Ash, 1987; ${ }^{5}$ Arens, 2001 (área aberta); ${ }^{6}$ Arens, 2001 (interior da mata); ${ }^{7}$ Seiler, 1981 e ${ }^{8}$ Chiou et al., 2001.

\begin{tabular}{lcl}
\hline \multicolumn{1}{c}{ Espécie } & $\begin{array}{c}\text { Número } \\
\text { Médio de } \\
\text { Frondes (ano) }\end{array}$ & Local de Estudo \\
\hline Cyathea atrovirens $^{1}$ & $7-14$ & $\begin{array}{l}\text { Campo Bom/RS } \\
\text { - Brasil }\end{array}$ \\
Cyathea atrovirens $^{2}$ & 8.6 & $\begin{array}{l}\text { Novo Hamburgo/ } \\
\text { RS - Brasil } \\
\text { Cyathea pubescens }\end{array}$ \\
Cyathea hornei $^{3}$ & 7 & Jamaica \\
Cyathea caracasana $^{5}$ & $3-11$ & Ilhas Fiji \\
Cyathea caracasana $^{6}$ & 21 & Colômbia \\
Alsophila salvinii $^{7}$ & 7 & Colômbia \\
Cibotium taiwanense $^{8}$ & 6 & El Salvador \\
Taiwan
\end{tabular}

Observamos uma relação negativa entre a taxa de crescimento relativo anual do cáudice e o tamanho do cáudice $\left(\mathrm{T}_{\mathrm{CR}}\right)\left(\mathrm{R}^{2}=-0,41 ; \mathrm{P}=0,043\right)$ (Figura 4). Maiores taxas de crescimento relativo de cáudice em indivíduos de menor tamanho são freqüentes em populações de pteridófitas arborescentes situadas no interior de florestas, uma vez que apresentar uma maior taxa de crescimento relativo significa aumentar as chances das plantas menores acessarem a luz no sub-bosque.

Os cáudices dos indivíduos analisados cresceram em média $0,70 \pm 0,21 \mathrm{~cm} \cdot \mathrm{mês}^{-1}$, com um crescimento máximo absoluto de $1,08 \mathrm{~cm} . \mathrm{mês}^{-1}$. Estes valores se aproximam da média verificada para uma população de Cyathea caracasana $\left(1,0 \mathrm{~cm} \cdot \mathrm{mês}^{-1}\right)$ crescendo em uma área aberta na Colômbia (Arens, 1998), estando muito acima dos valores observados para outras espécies ocorrendo no interior da mata (Ortega, 1984; Arens, 1998). Schmitt e Windisch (2006a) reportaram para uma população da mesma espécie, localizada em uma mata secundária, um crescimento médio anual de $2,48 \mathrm{~cm}^{-1}$. Provavelmente um maior crescimento dos cáudices no 
presente estudo pode ser explicado pela luminosidade, uma vez que a população estudada ocorre em uma área aberta, exposta a incidência solar plena.

Observações de campo mostram que cerca de $20 \%$ das espécies de pteridófitas em Zâmbia, ao menos ocasionalmente, sobrevivem ao fogo. Sua resistência ao fogo resulta de uma série de traços morfológicos e fisiológicos, que usualmente são reconhecidos como características das xerófitas (Kornás, 1993). Poucas pteridófitas estão aptas a persistir em ambientes sujeitos a intensas queimadas, sendo que somente algumas destas podem ser reconhecidas, devido a uma série de adaptações, como verdadeiras pirófitas (Kornás, 1978). Há indícios de que o rizoma das espécies classificadas como geófitas (ex. Pteridium aquilinum (L.) Kuhn e Rumohra adiantiformis (Forst.) Ching) atribuem a estas a capacidade de resistir a queimadas (C. R. Lehn observação pessoal).

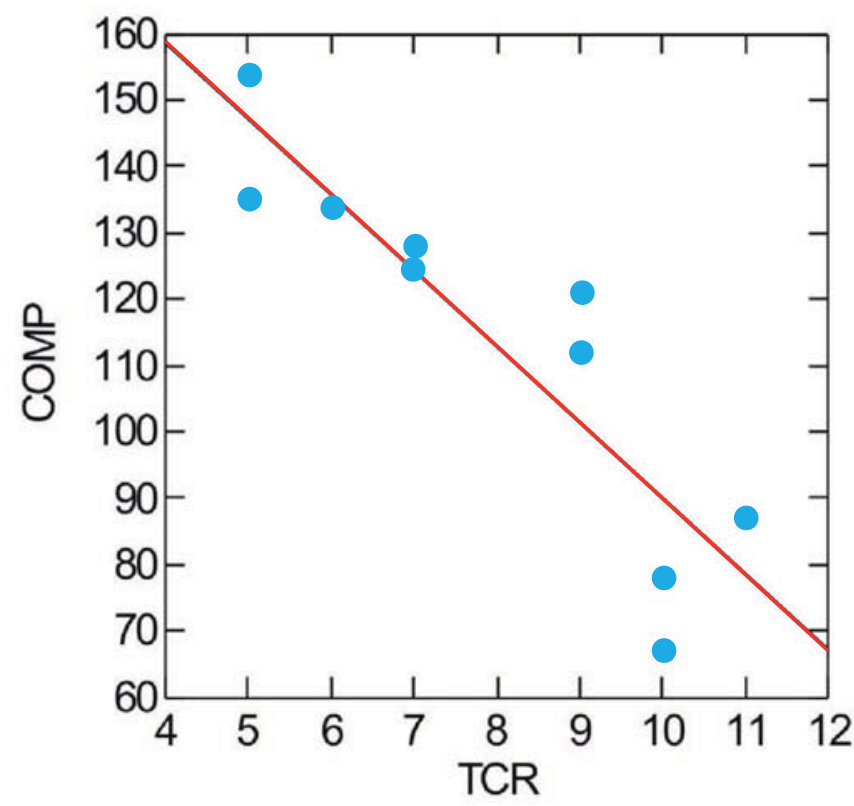

FIGURA 4: Relação entre comprimento do cáudice (COMP) e taxa anual de crescimento do cáudice $\left(\mathrm{T}_{\mathrm{CR}}\right)$, para uma população Cyathea atrovirens (Langsd. \& Fisch.) Domin, situada no município de Campo Bom/RS, Brasil.

A base dos pecíolos e a densa camada de raízes adventícias na base do cáudice de $C$. atrovirens podem contribuir de maneira significativa, para que os indivíduos atingidos pelo fogo não venham a perecer frente aos danos causados pela queimada. Considerando que a maioria dos indivíduos observados produziu frondes novas e férteis em um período de tempo relativamente curto (média $=25$ dias) após a queimada e que a taxa de produção de frondes foi superior ao observado por Schmitt e Windisch (2006a), podemos considerar $C$. atrovirens uma pteridófita capaz de suportar a passagem de fogo rápido. Tryon e Tryon (1982) reportam a ocorrência de C. atrovirens em áreas elevadas da Serra do Mar no sudeste brasileiro, destacando a sobrevivência de indivíduos em locais onde toda a cobertura do solo foi destruída pela ação de queimadas.

Os dados aqui obtidos são preliminares e podem servir de referência para futuras comparações com outras populações da referida espécie, contribuindo significativamente para a determinação da real capacidade de resiliência da mesma, após sofrer os impactos causados pelo fogo.

\section{Agradecimentos}

Os autores agradecem ao Dr. Erich Fischer pelas sugestões e críticas ao manuscrito; A CAPES pela bolsa de mestrado concedida aos autores.

\section{Referências}

Arens, N. C. 1998. Distribution of tree ferns (Cyatheaceae) across the successional mosaic in an Andean cloud forest, Nariño, Colombia. American Fern Journal, 88 (2): 60-71.

Arens, N. C. 2001. Variation in performance of the tree fern $C y$ athea caracasana (Cyatheaceae) across a successional mosaic in an Andean cloud forest. American Journal of Botany, 88 (3): 545 551.

Ash, J. 1987. Demography of Cyathea hornei (Cyatheaceae), a tropical tree fern in Fiji. Australian Journal of Botany, 35: 331342.

Chiou, W-L.; Lin, J-C.; Wang, J-Y. 2001. Phenology of Cibotium taiwanense (Dicksoniaceae). Taiwan Journal of Botany, 16 (4): 209-215.

Felfili, J. M.; Silva-Junior, M. C.; Rezende, A. V. 1999. Estudo fenológico de Stryphnodendron adstringens (Mart.) Coville no cerrado sensu stricto da Fazenda Água Limpa no Distrito Federal, Brasil. Revista Brasileira de Botânica, 22 (1): 11-16.

Fernandes, I. 1997. Taxonomia e fitogeografia de Cyatheaceae e Dicksoniaceae nas Regiões Sul e Sudeste do Brasil. Tese de Doutorado, Universidade de São Paulo, Brasil, 435pp.

Fernandes, I. 2000. Taxonomia dos representantes de Dicksoniaceae no Brasil. Pesquisas Botânica, 50: 5-26. 
Fernandes, I. 2003. Taxonomia dos representantes de Cyatheaceae do Nordeste Oriental do Brasil. Pesquisas Botânica, 54: 1-54.

Gignoux, J.; Clobert, J.; Menaut, J. C. 1997. alternative fire resistance strategies in savanna trees. Oecologia, 110 (4): 576-583.

Kornás, J. 1975. Tuber production and fire-resistance in Lycopodium carolinianum L. in Zambia. Acta Societatis Botaniae Polonae, 44: 653-663.

Kornás, J. 1978. Fire-Resistance in the Pteridophytes of Zambia. Fern Gazette, 11 (6): 373-384.

Kornás, J. 1979. Distribution and ecology of the pteridophytes in Zambia. Warszawa-Kraków, Pánstwowe Wydawnictwo Naukowe, Kraków, Polska, 205pp.

Kornás, J. 1993. The significance of historical factors and ecological preference in the distributions of African pteridophytes. Journal of Biogeography, 20: 281-286.

Lehn, C. R. 2008. Aspectos estruturais e fenológicos de uma população de Danaea sellowiana C. Presl (Marattiaceae) em uma Floresta Estacional Semidecidual no Brasil Central. Dissertação de Mestrado, Universidade Federal de Mato Grosso do Sul, Brasil, 92pp.

Lellinger, D. B. 1987. The disposition of Trichopteris (Cyatheaceae). American Fern Journal, 77 (3): 90-94.

Lorscheitter, M. L.; Ashraf, A. R.; Bueno, R. M.; Mosbruger, V. 1998. Pteridophyte Spores of Rio Grande do Sul Flora, Brazil. Part I. Palaeontographica, 246: 1-113.

Ortega, M. F. J. 1984. Notas sobre la autoecologia de Sphaeropteirs senilis (K1.) Tryon (Cyatheaceae) em el Parque Nacional El Ávila. Pittieria, 12: 31-53.

Ough, K.; Murphy, A. 2004. Decline in tree-fern abundance after clearfell harvesting. Forest Ecology and Management, 199: 153163.

Pinnard, M. A.; Huffman, J. 1999. Fire resistance and bark properties of trees in a seasonally dry forest in eastern Bolivia. Journal of Tropical Ecology, 13: 727-740.

Prado, J.; Freitas, C. A. A. 2005. Flora da Reserva Ducke, Amazonas, Brasil: Pteridophyta - Cyatheaceae. Rodriguésia, 56 (86): 35-37.

Ranal, M. 1995. Estabelecimento de pteridófitas em mata mesófila semidecídua do Estado de São Paulo. 3: Fenologia e Sobrevivência dos indivíduos. Revista Brasileira de Biologia, 55 (4): 777-787.

Schmitt, J. L. 2001. Desenvolvimento da fase esporofitica de Alsophila setosa Kaulf. (Pteridophyta, Cyatheaceae) em duas formações florestais no Estado do Rio Grande do Sul, Brasil. Dissertação Mestrado, Universidade do Vale do Rio dos Sinos, Brasil, 106pp.

Schmitt, J. L.; Windisch, P. G. 2003. Relação entre comprimento do estípite, produção de frondes e tamanho do cáudice, em Alsophila setosa Kaulf (Pteridophyta, Cyatheaceae). Pesquisas Botânica, 53: 55-64.

Schmitt, J. L.; Windisch, P. G. 2006a. Caracterização populacional e desenvolvimento da fase esporofítica de Cyathea atrovirens (Langsd. \& Fisch.) Domin (Cyatheaceae, Pteridophyta) no sul do Brasil. Libro de Resúmenes del IX Congreso Latinoamericano de Botánica, Santo Domingo, República Dominicana, p.628.

Schmitt, J. L.; Windisch, P. G. 2006b. Phenological aspects of frond production in Alsophila setosa (Cyatheaceae, Pteridophyta) in Southern Brazil. Fern Gazette, 17 (5): 263-270.

Sehnem, A. 1978. Ciateáceas. Flora Ilustrada Catarinense, Herbário Barbosa Rodrigues, Itajaí, Brasil, 115pp.

Seiler, R. L. 1981. Leaf turnover rates and natural history of the Central American tree fern Alsophila salvinii. American Fern Journal, 71: 75-81

Tanner, E. V. J. 1983. Leaf demography and growth of the tree fern Cyathea pubescens Mett. ex Kuhn in Jamaica. Botanical Journal of the Linnean Society, 87: 213-227.

Tryon, R. M. 1970. The classification of the Cyatheaceae. Contributions of Gray Herbarium, 200: 3-53

Tryon, R. M.; Tryon, A. F. 1982. Ferns and allied plants with special reference to Tropical America. Springer Verlag, New York, USA, $857 \mathrm{pp}$.

Windisch, P. G.; Tryon, R. M. 2001. The Serra Ricardo Franco (State of Mato Grosso, Brazil) as probable migration route and its present fern flora. Bradea, 8 (39): 267-276.

Wilkinson, L. 2004. Systat 11. Systat Software Inc, San José, USA, sem paginação.

Zar, J.H. 1999. Bioestatistical analysis. $4^{\text {th }}$ ed. Upper Saddle River, Prentice Hall, USA, 602pp. 